Pacific Journal of Mathematic 


\title{
THE NON-INVARIANCE OF HYPERBOLICITY IN PARTIAL DIFFERENTIAL EQUATIONS
}

\author{
H. H. JOHNSON
}

\begin{abstract}
Hyperbolicity is shown to be not an absolute invariant in the sense defined by the author. Specifically, an example of a nonhyperbolic system is given with a partial prolongation which is hyperbolic. A large class of systems is found which is closed under modified absolute equivalence and which contains all hyperbolic systems. These ideas are applied to give existence theorems for the initial value problem in several types of nonhyperbolic systems.
\end{abstract}

Since hyperbolicity is conveniently defined for quasi-linear systems, and as an additional reference, we define in $\S 1$ the ideas of partial prolongations and absolute equivalence for such systems. Since these problems and methods are generally local, we usually express them in coordinate notation. Ehresmann's jet notation could have been used to provide an invariant treatment. We also assume all manifolds and functions are infinitely differentiable, although it is not difficult to formulate the theorems for less smooth functions using available results in partial differential equations [3].

1. Definitions. Let $D^{p}$ and $D^{m}$ be open sets in $R^{p}=\left\{\left(x^{1}, \cdots, x^{p}\right)\right\}$ and $R^{m}=\left\{\left(z^{1}, \cdots, x^{m}\right)\right\}$, respectively.

Definition 1. A system $\Sigma$ on $D^{p} \times D^{m}$ is a system of functional and quasi-linear partial differential equations with $x^{1}, \cdots, x^{p}$ as independent and $z^{1}, \cdots, z^{m}$ as dependent variables:

$$
\begin{array}{ll}
f^{\alpha}\left(x^{1}, \cdots, x^{p}, z^{1}, \cdots, z^{m}\right)=0, & \alpha=1, \cdots, \alpha_{1}, \\
L^{\varsigma}=A_{\lambda}^{\beta^{i}} \frac{\partial z^{\lambda}}{\partial x^{i}}+B^{\beta}=0, & \beta=1, \cdots, \beta_{1},
\end{array}
$$

(we use the summation convention), where $f^{\alpha}, A_{\lambda}^{\beta^{i}}$ and $B^{\beta}$ are (infinitely differentiable) functions on $D^{p} \times D^{m}$. It is also required that the equations

$$
\begin{aligned}
f^{\alpha},{ }_{x^{i}} \frac{\partial z^{\lambda}}{\partial x^{i}}+f^{\alpha},{ }_{x^{i}}=0, \quad \alpha & =1, \cdots, \alpha_{1} \\
i & =1, \cdots, p,
\end{aligned}
$$

be a consequence of $L^{\beta}=0$. (We use the notation $f^{\alpha}{ }_{{ }_{2} \lambda}=\partial f^{\alpha} / \partial z^{\lambda}$, etc). 
Definition 2. If $\Sigma$ is the system in Definition 1 , its total prolongation $P \Sigma$ is the system on $D^{p} \times D^{m} \times R^{p m}=\left\{\left(x^{i}, z^{\lambda}, p_{j}^{\mu}\right) \mid i, j=\right.$ $1, \cdots, p ; \lambda, \mu=1, \cdots, m\}$ generated by the equations of $\Sigma$ together with

$$
\begin{aligned}
& \frac{\partial z^{\lambda}}{\partial x^{i}}-p_{i}^{\lambda}=0, \quad \frac{\partial p_{i}^{\lambda}}{\partial x^{j}}-\frac{\partial p_{j}^{\lambda}}{\partial x^{i}}=0, \\
& A_{\lambda}^{\beta_{\lambda}^{i}} \frac{\partial p_{i}^{\lambda}}{\partial x^{j}}+A_{\lambda}^{\beta^{i}},{ }_{{ }^{\mu}} p_{j}^{\mu} p_{i}^{\lambda}+A_{\lambda}^{\beta_{2}^{i},}{ }_{x j} p_{i}^{\lambda} \\
& \quad+B^{\beta},{ }_{x} p_{j}^{\lambda}+B^{\beta},{ }_{x^{j}}=0, \\
& \lambda, \mu=1, \cdots, m ; i, j=1, \cdots, p ; \beta=1, \cdots, \beta_{1} .
\end{aligned}
$$

Definition 3. A transform or change variables of is a diffeomorphism of the form

$$
\begin{aligned}
& \bar{z}^{\lambda}=\bar{\varphi}^{\lambda}\left(x^{1}, \cdots, x^{p}, z^{1}, \cdots, z^{m}\right) \\
& x^{i}=\bar{\psi}^{i}\left(x^{1}, \cdots, x^{p}\right)
\end{aligned}
$$

with inverse defined by $\varphi^{\lambda}, \psi^{i}$. Then $\Sigma$ is transformed according to the usual rules:

$$
\frac{\partial \bar{z}^{\lambda}}{\partial \bar{x}^{i}}=\left(\bar{\varphi}^{\lambda},{ }_{z^{\mu}} \frac{\partial z^{\mu}}{\partial x^{k}}+\bar{\varphi}^{\lambda},{ }_{x^{k}}\right) \psi^{k},{ }_{x^{i}} \cdot
$$

Thus, $D^{p} \times D^{m}$ is regarded as a fibre space over $D^{p}$.

Definition 4. A system $\Sigma_{1}$ on $D^{p} \times D^{m} \times D^{n}=\left\{\left(x^{i}, z^{\lambda}, u^{r}\right) \mid i=\right.$ $1, \cdots, p ; \lambda=1, \cdots, m ; \gamma=1, \cdots, n\}$ is a partial prolongation of the system $\Sigma$ in Definition 1 when it is generated by equations equivalent to those in $\Sigma$ together with equations of the form

$$
\begin{aligned}
& u^{\gamma}-C_{\lambda}^{\gamma j} \frac{\partial z^{\lambda}}{\partial x^{j}}-D^{\gamma}=0, \quad \gamma=1, \cdots, n, \\
& P^{\varsigma}=E_{r}^{\delta i} \frac{\partial u^{\gamma}}{\partial x^{i}}+F_{r}^{\delta i} \frac{\partial z^{\lambda}}{\partial x^{i}}+G^{\delta}=0, \quad \delta=1, \cdots, \delta_{1},
\end{aligned}
$$

where $C_{\lambda}^{\gamma j}, D^{r}$ are functions on $D^{p} \times D^{m}$. It is further required that if $P \Sigma$ is the total prolongation of $\Sigma$ as in Definition 2, then

$$
\begin{aligned}
E_{\gamma}^{\delta i}\left(C_{\lambda}^{\gamma j} \frac{\partial p_{j}^{\lambda}}{\partial x^{i}}\right. & +C_{\lambda}^{\gamma j},{ }_{{ }^{\mu}}{ }^{\mu} p_{j}^{\lambda} p_{i}^{\mu}+C_{\lambda}^{\gamma j}{ }_{{ }_{i}} p_{j}^{\lambda} \\
& \left.+D^{\gamma},{ }_{2}{ }_{\lambda} p_{i}^{\lambda}+D^{\gamma},{ }_{x^{i}}\right)+F_{\lambda}^{\delta i} p_{i}^{\lambda}+G^{\delta}=0
\end{aligned}
$$

are to be consequences of the equations in $P \Sigma$. When $C_{\lambda}^{\gamma j}=0, \Sigma$ is 
called an admissible restriction of $\Sigma_{1}$.

Observe that $P \Sigma$ is itself a partial prolongation of $\Sigma$. If $z^{\lambda}=$ $\bar{z}^{\lambda}\left(x^{1}, \cdots, x^{p}\right)$ is a solution of $\Sigma$ then $p_{i}^{\lambda}=\partial z^{\lambda} / \partial x^{i}$ define a solution of $P \Sigma$, and hence $u^{\gamma}=C_{\lambda}^{\gamma j}(x, \bar{z})\left(\partial z^{\lambda} / \partial x^{j}\right)+D^{r}(x, \bar{z})$ define a solution of $\Sigma_{1}$. The above definitions are somewhat more restrictive than in [2], but they are essentially equivalent. In [2] it was shown that if $\Sigma_{1}$ is a partial prolongation of $\Sigma$, then $P \Sigma$ is a partial prolongation of $\Sigma_{1}$.

DEFINITION 5. If $\Sigma_{0}, \Sigma_{1}, \cdots, \Sigma_{n}$ is a finite sequence of systems such that for every $i=1, \cdots, n, \Sigma_{i}$ is a partial prolongation or transform of $\Sigma_{i-1}$ or else $\Sigma_{i-1}$ is a partial prolongation or transform of $\Sigma_{i}$, then $\Sigma_{0}$ and $\Sigma_{n}$ are absolutely equivalent.

2. Hyperbolicity. We now treat $D^{p}$ as a product space $D^{p-1} \times$ $D^{1}=\left\{\left(x^{1}, \cdots, x^{p-1}\right) \times\left(x^{p}\right)\right\}$ and discuss systems "hyperbolic in the $x^{p}$ direction." Any change of variables must preserve this product structure on $D^{p}$.

Notation. If $F\left(x^{i}, z^{\lambda}, \partial z^{\mu} / \partial x^{j}\right)$ is any first-order partial differential function, denote by $F$; ${ }_{j}$ the second-order function

$$
F ;_{j}=F,{ }_{x^{j}}+F,{ }_{z^{j}} \frac{\partial z^{\lambda}}{\partial x^{j}}+F,{ }_{\left(\partial z^{\lambda} / \partial x^{i}\right)} \frac{\partial^{2} z^{\lambda}}{\partial x^{i} \partial x^{j}} .
$$

(Note that $F$ and $F ;{ }_{j}$ may be regarded as functions on spaces of jets. We always regard $\partial^{2} z^{\lambda} / \partial x^{i} \partial x^{j}=\partial^{2} z^{\lambda} / \partial x^{j} \partial x^{i}$.)

Definition 6. The system $\Sigma$ is involutive in the $x^{p}$-direction if it is generated by equations of the form

$$
\begin{aligned}
& f^{\alpha}\left(x^{1}, \cdots, x^{p}, z^{1}, \cdots, z^{m}\right)=0, \quad \alpha=1, \cdots, \alpha_{1}, \\
& M^{\lambda}=\frac{\partial z^{\lambda}}{\partial x^{p}}-\sum_{a=1}^{p=1} A_{\mu}^{\lambda a} \frac{\partial z^{\mu}}{\partial x^{a}}-B^{\lambda}=0, \quad \lambda=1, \cdots, m, \\
& L^{\beta}=\sum_{a=1}^{p-1} C_{\mu}^{\beta a} \frac{\partial z^{\mu}}{\partial x^{a}}+D^{\beta}=0, \quad \beta=1, \cdots, \beta_{1} .
\end{aligned}
$$

It is required that

$$
\begin{aligned}
L^{\beta} ;{ }_{p} & -\sum_{a=1}^{p-1}\left(R_{\gamma}^{\beta a} L^{\gamma} ;_{a}+S_{\lambda}^{\beta a} M^{\lambda} ;_{a}\right) \\
& -T_{\gamma}^{\beta} L^{\gamma}-U_{\lambda}^{\beta} M^{\lambda}=0 \quad\left(\bmod f^{1}, \cdots, f^{\alpha_{1}}\right),
\end{aligned}
$$

where the $R_{\gamma}^{\beta a}, T_{\gamma}^{\beta}$ and $U_{\lambda}^{\beta}$ are functions of $x^{i}, z^{\lambda}, M^{\lambda}$ and $M^{\lambda}{ }_{a}, a=$ $1, \cdots, p-1$. (In the following the indices $a, b$ will run over $1, \cdots$, $p-1$ unless noted otherwise, $i, j=1, \cdots, p ; \lambda, \mu=1, \cdots, m$.) 
The equations $M^{2}=0$, are called the primary equations of while equations (1) are the secondary equations.

Involutiveness is not in general preserved under partial prolongations, since, in the notation of Definition 4 , no $P^{\delta}$ need occur. We shall usually be concerned with invariants among systems involutive in the $x^{p}$-direction. By a calculation it can be seen that this concept is preserved under a change of coordinates which leaves the product structure on $D^{p}$ invariant. We introduce the following finer equivalence relation.

Definition 7. Two systems involutive in the $x^{p}$-direction are $x^{p}$-equivalent if they are absolutely equivalent by means of a sequence of systems $\Sigma_{0}, \Sigma_{1}, \cdots, \Sigma_{n}$ where each $\Sigma_{i}$ is involutive in the $x^{p}$-direction.

Definition 8. Let $\Sigma$ be involutive in the $x^{p}$-direction. Suppose (in the notation of Definition 6) the equations of $\Sigma$ can be arranged so that the matrices $A_{\mu}^{\lambda a}$ and $R_{\gamma}^{\beta a}$ have the following property: there exist nonsingular real matrices $V_{\mu}^{\lambda}\left(x, z, \xi_{1}, \cdots, \xi_{p-1}\right)$ and $W_{r}^{\beta}\left(x, z, \xi_{1}, \cdots\right.$, $\left.\xi_{p-1}\right)$ on $D^{p} \times D^{m} \times R^{p-1}$ with inverses $\left(V^{-1}\right)_{\mu}^{\lambda}$ and $\left(W^{-1}\right)_{i}^{\beta}$ such that on $D^{p} \times D^{m} \times R^{p-1}$,

$$
V_{\mu}^{\lambda} A_{v}^{\mu a} \xi_{a}\left(V^{-1}\right)_{\omega}^{\nu}
$$

and

$$
W_{\gamma}^{\beta} R_{\delta}^{\gamma a} \xi_{a}\left(W^{-1}\right)_{\varepsilon}^{\delta}
$$

are diagonal matrices. Then $\Sigma$ is hyperbolic in the $x^{p}$-direction.

By a calculation it can be seen that this concept is preserved under an allowable change of variables.

THEOREM 1. Let $\Sigma$ be hyperbolic in the $x^{p}$-direction on a neighborhood of $\left(x_{0}, z_{0}\right)$. Using the notation of Definition 6, let

$$
z^{\lambda}=\varphi^{\lambda}\left(x^{1}, \cdots, x^{p-1}\right), \quad \lambda=1, \cdots, m,
$$

be defined on a neighborhood of $\left(x_{0}^{a}\right) \in D^{p-1}$ and satisfy

$$
\begin{aligned}
& \varphi^{\lambda}\left(x_{0}^{a}\right)=z_{0}^{\lambda}, \quad \lambda=1, \cdots, m, \\
& f^{\alpha}\left(x^{1}, \cdots, x^{p-1}, x_{0}^{p}, \varphi^{1}, \cdots, \varphi^{m}\right)=0, \quad \alpha=1, \cdots, \alpha_{1},
\end{aligned}
$$

and

$$
C_{\mu}^{\beta a}\left(x^{b}, x_{0}^{p}, \varphi^{\lambda}\right) \varphi^{\prime \prime},{ }_{x^{i}}+D^{\beta}\left(x^{b}, x_{0}^{p}, \varphi^{\lambda}\right)=0, \quad \beta=1, \cdots, \beta_{1} .
$$

Then there exists a unique solution $z^{\lambda}=F^{\lambda}\left(x^{1}, \cdots, x^{p}\right)$ of ¿ on a neighborhood of $\left(x_{0}^{i}\right)$ satisfying 


$$
F^{\lambda}\left(x^{1}, \cdots, x^{p-1}, x_{0}^{p}\right)=\varphi^{\lambda}\left(x^{1}, \cdots, x^{p-1}\right), \quad \lambda=1, \cdots, m .
$$

Proof. Apply standard existence theorems [3] to solve $M^{\lambda}=0$ with the given initial conditions. This solution is unique. The functions $L^{\beta}$ satisfy a second system of linear hyperbolic equations with zero initial values. By uniqueness, the $L^{\beta}$ are zero. Similar arguments show the $f^{\alpha}$ to be zero when evaluated at the solution of $M^{\lambda}=0$.

ExAmPle. The system

$$
M^{1}=\frac{\partial z^{1}}{\partial x^{2}}=0, \quad M^{2}=\frac{\partial z^{2}}{\partial x^{2}}-\frac{\partial z^{2}}{\partial x^{1}}=0, \quad M^{3}=\frac{\partial z^{3}}{\partial x^{2}}-\frac{\partial z^{1}}{\partial x^{1}}=0
$$

is involutive in the $x^{2}$-direction, but its matrix $A_{\mu}^{\lambda_{1}}$, having eigenvalues $0,0,1$ and rank 2, is not diagonalizable. The partial prolongation $\Sigma_{1}$ obtained by adding $\left(\partial z^{1} / \partial x^{1}\right)-u^{1}=0$ and $\left(\partial u^{1} / \partial x^{2}\right)=0$ is, however, hyperbolic in the $x^{2}$-direction, for this larger system may be written

$$
\begin{aligned}
& \frac{\partial z^{1}}{\partial x^{2}}=0, \quad \frac{\partial z^{2}}{\partial x^{2}}-\frac{\partial z^{2}}{\partial x^{1}}=0, \quad \frac{\partial z^{3}}{\partial x^{2}}-u^{1}=0, \\
& \frac{\partial u^{1}}{\partial x^{2}}=0, \quad \frac{\partial z^{1}}{\partial x^{1}}-u^{1}=0 \text {. }
\end{aligned}
$$

The matrix $A_{\mu}^{\lambda_{1}}$ is now diagonalized while $R_{\gamma}^{\beta 1}$ is 1 -dimensional, hence diagonal.

This example shows hyperbolicity is not invariant under partial prolongations, even if both systems are involutive in the $x^{p}$-direction. Yet the initial value problem can be solved for $\Sigma$ in the example. Given $\varphi^{\lambda}\left(x^{1}\right)$ as initial functions, let $\psi^{1}=\partial \varphi^{1} / \partial x^{1}$ be an initial function for $u^{1}$ in $\Sigma_{1}$. Solving $\Sigma_{1}$ will yield a solution of $\Sigma$.

THEOREM 2. Let $\Sigma$ be $x^{p}$-equivalent to $\Sigma_{1}$ which is hyperbolic in the $x^{p}$-direction. Then given the initial conditions of Theorem 1 for $\Sigma$ a solution may be found as in Theorem 1.

Proof. It suffices to consider one pair at a time in the sequence of systems joining $\Sigma$ and $\Sigma_{1}$, showing that initial conditions carry over naturally and recalling that any solution of one system induces a solution of the others. Thus, one need consider only single partial prolongations or changes of variables. In each case the result follows from a detailed calculation.

3. Complex systems. In this section we determine a class of systems which contains the hyperbolic systems and is closed under $x^{p}$-equivalence. 
Definition 9. A system which is involutive in the $x^{p}$-direction is said to be $x^{p}$-complex on an open set $U \subset D^{p} \times D^{m}$ if, in the notation of Definition 6,

(1) for every choice of variables $x^{1}, \cdots, x^{p-1}$ and

(2) for every choice of functions $H_{\beta}^{\lambda}$ on $U$, the matrix $A_{\mu}^{\lambda}+H_{\beta}^{\lambda} C_{\mu}^{\beta 1}$ always has at least one nonreal eigenvalue at each point of $U$.

Hyperbolic systems are not $x^{p}$-complex since in the notation of Definition 7, with $H_{\beta}^{\lambda}=0$ and $\xi_{a}=\delta_{a}^{1}, A_{\mu}^{\lambda 1}$ is (real) diagonalizable. We shall show that $x^{p}$-complex systems form a class which is closed under $x^{p}$-equivalence. The complimentary class contains all systems hyperbolic in the $x^{p}$-direction.

Lemma 1. Let $A$ be an $m \times m$ matrix of functions on an open set $\mathrm{U} \subset D^{p} \times D^{m}$. Let

$$
C=\left(\begin{array}{l}
V \\
0
\end{array}\right)
$$

be an $m \times r$ matrix of functions on $\bigcup$ where $V$ is $s \times r$ of rank $s$. Assume that for every $r \times m$ matrix function $H$ on $\mathbf{U}, A+C H$ has at least one nonreal eigenvalue at each point of $\mathrm{U}$. Then $s \leqq m-2$ and there exists on a neighborhood of each point in $\mathbf{U}$ a nonsingular matrix of functions of the form

$$
P=\left(\begin{array}{ll}
I & 0 \\
0 & P_{1}
\end{array}\right)
$$

where $I$ is $s \times s$ identity such that

$$
P A P^{-1}=\left(\begin{array}{cc}
W & X \\
0 & A_{1}
\end{array}\right)
$$

where $A_{1}$ is $2 \times 2$ with nonreal eigenvalues. The converse is also true.

Proof. The converse follows immediately. The lemma may be proved by induction on $m \geqq 2$. If $m=2$, then $s$ is 0,1 or 2 . If $s=0$, then $C=0$, hence $A=C H=A=A_{1}$. When $s=1$ or 2 the other hypotheses cannot be fulfilled.

Now assume the lemma true for all matrices $A$ of order $<m$. Given any $R^{\prime}$ of order $s \times s$ and $R^{\prime \prime}$ of order $s \times(m-s)$ one may choose $H$ such that

$$
A+C H=\left(\begin{array}{cc}
R^{\prime} & R^{\prime \prime} \\
A^{\prime} & A^{\prime \prime}
\end{array}\right)
$$


Then if $A+C H$ is to have always a nonreal eigenvalue, certainly $s \leqq m-2$. On a neighborhood $\mathbf{U}^{\prime}$ of any point in $\mathbf{U}$ a nonsingular $(m-s) \times(m-s)$ matrix function $P_{1}$ may be found so that

$$
P_{1} A^{\prime}=\left(\begin{array}{c}
V^{\prime} \\
0
\end{array}\right)=C^{\prime}
$$

where $V^{\prime}$ is $t \times(m-s)$ and has rank $t$. Then

$$
\left(\begin{array}{ll}
I & 0 \\
0 & P_{1}
\end{array}\right)\left(\begin{array}{ll}
R^{\prime} & R^{\prime \prime} \\
A^{\prime} & A^{\prime \prime}
\end{array}\right)\left(\begin{array}{ll}
I & 0 \\
0 & P_{1}^{-1}
\end{array}\right)=\left(\begin{array}{ll}
R^{\prime}, & R^{\prime} P^{-1} \\
C, & P_{1} A^{\prime \prime} P_{1}^{-1}
\end{array}\right)
$$

has at least one nonreal eigenvalue for every $R^{\prime}, R^{\prime \prime}$. Then so does

$$
\left(\begin{array}{cc}
I-K \\
0 & I
\end{array}\right)\left(\begin{array}{ll}
R^{\prime}, & R^{\prime \prime} P_{1}^{-1} \\
C^{\prime}, & P_{1} A^{\prime \prime} P_{1}^{-1}
\end{array}\right)\left(\begin{array}{cc}
I & K \\
0 & I
\end{array}\right)
$$

for every choice of $K$.

In particular, if $R^{\prime}=K C^{\prime}, R^{\prime \prime}=K P_{1} A^{\prime \prime}+K C^{\prime} P_{1}-R^{\prime} K P_{1}$, it follows that $P_{1} A^{\prime \prime} P_{1}^{-1}+C^{\prime} K$ has nonreal eigenvalues for every choice of $K$. The induction hypothesis applies to $P_{1} A^{\prime \prime} P_{1}^{-1}$ and $C^{\prime}$, so on a neighborhood of each point in $\mathrm{U}^{\prime} \subset \mathrm{U}$, there is a matrix $P_{2}$ such that

$$
\left(\begin{array}{ll}
I & 0 \\
0 & P_{2}
\end{array}\right) P_{1} A^{\prime \prime} P_{1}^{-1}\left(\begin{array}{cc}
I & 0 \\
0 & P_{2}^{-1}
\end{array}\right)=\left(\begin{array}{cc}
W^{\prime} & X^{\prime} \\
0 & A_{2}
\end{array}\right)
$$

where $A_{2}$ is $2 \times 2$ with nonreal eigenvalues, $I$ is $t \times t$ and $t \leqq m-$ $s-2$. Now take

$$
P=\left(\begin{array}{ll}
I_{2} & 0 \\
0 & P_{2}
\end{array}\right)\left(\begin{array}{ll}
I_{1} & 0 \\
0 & P_{1}
\end{array}\right)
$$

where $I_{1}$ is the $s \times s$ and $I_{2}$ is the $(s+t) \times(s+t)$ identity matrix.

THEOREM 3. Let $\Sigma_{1}$ be a partial prolongation of $\Sigma$ where both are involutive in the $x^{p}$-direction. If $\Sigma$ is $x^{p}$-complex on $\mathbf{U} \subset D^{p} \times$ $D^{m}$, then $\Sigma_{1}$ is $x^{p}$-complex on $\mathbf{U} \times R^{n}$. If $\Sigma_{1}$ is $x^{p}$-complex on $\mathbf{U} \times \boldsymbol{V}$ where $\mathrm{U}$ is open in $D^{p} \times D^{m}$, then $\Sigma$ is $x^{p}$-complex on $\mathrm{U}$.

Proof. Let $\Sigma$ have the form in Definition 6 while $\Sigma_{1}$ contains the additional equations

$$
\begin{aligned}
& \cup^{\pi}-E_{\lambda}^{\pi a} \frac{\partial z^{\lambda}}{\partial x^{a}}-F^{\pi}=0, \\
& \frac{\partial u^{\pi}}{\partial x^{p}}-G_{\lambda}^{\pi a} \frac{\partial z^{\lambda}}{\partial x^{a}}-H_{\rho}^{\pi a} \frac{\partial u^{\rho}}{\partial x^{a}}-I^{\pi}=0, \\
& J_{\pi}^{\gamma a} \frac{\partial u^{\pi}}{\partial x^{a}}+K_{\lambda}^{\gamma 2} \frac{\partial z^{\lambda}}{\partial x^{a}}+N^{\gamma}=0,
\end{aligned}
$$


$\pi, \rho=1, \cdots, n ; \gamma=1, \cdots, \gamma_{1} . \quad$ (Observe that $\partial z^{\lambda} / \partial x^{\rho}$ can be eliminated using equations in $\Sigma$.) Definition 4 requires that the equations obtained by replacing $\partial u^{\pi} / \partial x^{i}$ by expressions in $p_{i}^{\lambda}$ and $\partial p_{i}^{\lambda} / \partial x^{j}$ must occur in $P \Sigma$. Considering the coefficient of $\partial p_{i}^{\lambda} / \partial x^{1}$ in these expressions one sees that for some functions $N_{\beta}^{\gamma}$ and $M_{\beta}^{\pi}$ of $x^{i}, z^{\lambda}$,

$$
J_{\pi}^{\gamma 1} E_{\lambda}^{\pi 1}=N_{\beta}^{\gamma} C_{\lambda}^{\beta 1} .
$$

and

$$
E_{\lambda}^{\tau 1} A_{\eta^{\prime \prime}}^{\lambda_{1}}-H_{\rho}^{\tau 1} E_{\lambda}^{\rho_{1}}=M_{\beta}^{\pi} C_{\lambda}^{\beta_{1}} .
$$

Thus, if $J=\left(J_{\pi}^{r 1}\right), E=\left(E_{\hat{\lambda}}^{\pi 1}\right)$, etc.,

$$
E J=C N \text { and } A E-E H=C M .
$$

Now assume $\Sigma$ is $x^{p}$-complex, so for every choice of $x^{1}, \cdots, x^{p-1}$ and $M_{0}, A+C M_{0}$ has a nonreal eigenvalue at each point of $\mathrm{U}$. To prove that $\Sigma_{1}$ is $x^{p}$-complex we must show that

$$
\left(\begin{array}{cc}
A & G \\
0 & H
\end{array}\right)+\left(\begin{array}{ccc}
C & E & K \\
0 & 0 & J
\end{array}\right)\left(\begin{array}{cc}
M_{1} & M_{2} \\
M_{3} & M_{4} \\
M_{5} & M_{6}
\end{array}\right)=Z
$$

has a nonreal eigenvalue for every choice of $M_{1}, \cdots, M_{6}$, where $G=$ $\left(G_{\lambda}^{\pi 1}\right), H=\left(H^{\pi 1}\right)$ etc.

On a neighborhood of $\left(x_{0}, z_{0}\right) \in \mathbf{U}$ let $\bar{P}$ be an $m \times m$ nonsingular matrix of functions so that

$$
\bar{P} C=\left(\begin{array}{l}
V \\
0
\end{array}\right)=\bar{C}
$$

where $V$ is $s \times r$ and of rank $s \leqq m-2$. Let $\bar{A}=\bar{P} A \bar{P}^{-1}$. By Lemma 1 there is a nonsingular $P$ on a neighborhood of $\left(x_{0}, z_{0}\right)$ such that $P \bar{C}=\bar{C}$ and

$$
P \bar{A} P^{-1}=\left(\begin{array}{cc}
W & X \\
0 & A_{1}
\end{array}\right)
$$

where $A_{1}$ is $2 \times 2$ with nonreal eigenvalues. This $P$ can be chosen so that

$$
A_{1}=\left(\begin{array}{rr}
a & b \\
-b & a
\end{array}\right)
$$

Then the complex vectors

$$
q_{1}=(0, \cdots, 0,1, i), \quad r_{1}=(0, \cdots, 0,1,-i)
$$


satisfy

$$
q_{1} P \bar{A} P^{-1}=(a-i b) q_{1}, r_{1} P \bar{A} P^{-1}=(a+i b) r_{1},
$$

while

$$
q_{1} P \bar{C} M_{0} P^{-1}=r_{1} P \bar{C} M_{0} P^{-1}=0 .
$$

Hence $q=q_{1} P \bar{P}$ and $r=r_{1} P \bar{P}$ are complex eigenvectors of $A$ which are carried to zero by $C$.

Since $A E-E H=C M, q E$ is an eigenvector of $H$ belonging to the eigenvalue $a-i b$ while $r E$ belongs to $a+i b$. From $E J=C N$ it follows that $(q E) J=(r E) J=0$. For every $M_{6}, H+J M_{6}$ has these same eigenvectors and values. Letting $Q$ be $n \times n$ nonsingular so that

$$
\bar{Q} J=\left(\begin{array}{c}
J^{\prime} \\
0
\end{array}\right)
$$

where $J^{\prime}$ is $t \times U$ with rank $t<n-2$, there must exist by Lemma 1 a matrix $Q_{1}$ on a neighborhood of $\left(x_{0}, z_{0}\right)$ such that

$$
Q_{1}\left(\bar{Q} H \bar{Q}^{-1}\right) Q_{1}^{-1}=\left(\begin{array}{cc}
W^{\prime} & X^{\prime} \\
0 & H_{1}
\end{array}\right)
$$

where $H_{1}$ is $2 \times 2$ with nonreal eigenvalues. Letting $Q=Q_{1} \bar{Q}$, it follows that

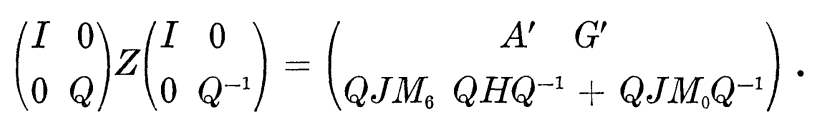

The lower two rows in this matrix are $\left(0 H_{1}\right)$. It follows that for every choice of $M_{1}, \cdots, M_{6}$, the matrix $Z$ has nonreal eigenvalues for any $\left(x_{0}, z_{0}\right) \in \mathbf{U},\left(u^{1}, \cdots, u^{n}\right) \in R^{n}$.

The converse follows at once by choosing $M_{2}=\cdots=M_{6}=0$ in $Z$.

4. Examples. Several nonanalytic systems for which the initial value problem may be solved are $x^{p}$-equivalent to systems hyperbolic in the $x^{p}$-direction.

Theorem 4. The system

$$
\frac{\partial z}{\partial x^{p}}=f\left(x^{1}, \cdots, x^{p}, z, \frac{\partial z}{\partial x^{1}}, \cdots, \frac{\partial z}{\partial x^{p-1}}\right)
$$

is absolutely equivalent to a system hyperbolic in the $x^{p}$-direction. (We have not included such general systems is our definitions, but 
these can be extended in an obvious way).

Proof. The total prolongation is obtained by adding

$$
\begin{aligned}
& M_{i}=\partial z / \partial x^{i}-q_{i}, \quad L_{i j}=\partial q_{i} / \partial x^{j}-\partial q_{j} / \partial x^{i}, \\
& N_{2}=\partial q_{p} / \partial x^{i}-f,{ }_{x^{i}}-f,{ }_{z} q_{i}-f,{ }_{q_{a}}\left(\partial q_{i} / \partial x^{a}\right)=0, \\
& i, j=1, \cdots, p ; \alpha=1, \cdots, p-1 .
\end{aligned}
$$

Then $q_{p}-f\left(x, z, q_{1}, \cdots, q_{p-1}\right)=0$ is the 0 -order equation; $M_{p}=0$, $L_{a p}=0$ and $N_{p}=0$ are the primary equations, and $M_{a}=0, L_{a b}=0$, $N_{a}=0$ generate the secondary equations. Since

$$
\begin{aligned}
L_{a b ; p}= & L_{p a ; b}-L_{p b ; a} \\
M_{a ; p}= & M_{p ; a}-L_{a p}, \\
N_{a ; p}= & N_{p ; a}+M_{a}\left(f,{ }_{x}{ }^{p, z}+f,{ }_{z},{ }_{a} q p-f,{ }_{q_{b}},{ }_{a} L_{b p}\right)-f,{ }_{a} L_{a p} \\
& -L,{ }_{q_{a}},{ }_{a} L_{b p}-f,{ }_{{ }_{q}},{ }_{q c} L_{c p} \frac{\partial q_{b}}{\partial x^{a}} \\
& -f, q_{b} L_{p b ; a}
\end{aligned}
$$

the secondary equations have matrix $R_{\gamma}^{\beta a}=0$, in the notation of Definition 6 . The primary equations may be arranged in the form

$$
\begin{aligned}
& M_{p}=0, \\
& L_{a p}+N_{a}=\frac{\partial q_{a}}{\partial x^{p}}-f,{ }_{q_{b}} \frac{\partial q_{a}}{\partial x^{b}}-f,{ }_{x} a-f,{ }_{q} q_{a}=0, \\
& N_{p}=0,
\end{aligned}
$$

with relevant matrix $A_{\mu}^{\lambda b}=\delta_{\mu}^{\lambda} f,{ }_{q_{b}}$. For any $\xi_{1}, \cdots, \xi_{p-1}, A_{\mu}^{\lambda b} \xi_{b}$ is diagonal.

The example in $\S 2$ can be generalized in the following

THEOREM 5. If the system $\Sigma$ on $D^{2} \times D^{m}$

$$
\begin{aligned}
& M^{\lambda}=\frac{\partial z^{\lambda}}{\partial x^{2}}-A_{\mu}^{\lambda} \frac{\partial z^{\mu}}{\partial x^{1}}-B^{\lambda}=0, \quad \lambda=1, \cdots, m, \\
& L^{\beta}=C_{\lambda}^{\beta} \frac{\partial z^{\lambda}}{\partial x^{1}}+D^{\beta}=0, \quad \beta=1, \cdots, \beta_{1},
\end{aligned}
$$

is involutive in the $x^{2}$-direction and satisfies

(1) $A_{\mu}^{\lambda}, B^{\lambda}$ are constants,

(2) $A_{\mu}^{\lambda}$ has only real eigenvalues and elementary divisors of degree at most 2 ,

(3) the auxiliary system is hyperbolic, then $\Sigma$ is $x^{2}$-equivalent to a system hyperbolic in the $x^{2}$-direction. 
Proof. Let $P=\left(v_{\mu}^{\lambda}\right)$ be a nonsingular constant matrix with inverse $P^{-1}=\left(w_{\mu}^{\lambda}\right)$ so that $P^{-1} A P=\operatorname{diag}\left(J_{1}, \cdots, J_{r}, \lambda^{r+1}, \cdots, \lambda^{n}\right)=J$ where

$$
J_{k}=\left(\begin{array}{cc}
\lambda^{k} & 1 \\
0 & \lambda^{k}
\end{array}\right)
$$

Then $A P=P J$, so

$$
A_{\lambda}^{\mu} v_{\mu}^{2 k-1}=\lambda^{1} v_{\lambda}^{2 k-1}, \quad k=1, \cdots, r .
$$

Consider the partial prolongation $\Sigma_{1}$ on $D^{2} \times D^{m} \times R^{k}$ with new variables $u^{1}, \cdots, u^{k}$ obtained by adding to $\Sigma$ the equations

$$
\begin{array}{ll}
I^{\theta}=\frac{\partial u^{\theta}}{\partial x^{2}}-\lambda^{\theta} \frac{\partial u^{\theta}}{\partial x^{1}}=0 & \text { (no summation) } \\
K^{\theta}=u^{\theta}-v_{\lambda}^{2 \theta-1} \frac{\partial z^{\lambda}}{\partial x^{1}}, & \theta=1, \cdots, k .
\end{array}
$$

It is not difficult to check that $\Sigma_{1}$ is a partial prolongation. Since $K^{\theta} ; \hat{\imath}=I^{\theta}+\lambda^{\theta} K^{\theta} ; \hat{1}-v_{\lambda}^{2 \theta-1} M^{\lambda} ; \hat{1}$, (no summation on $\theta$ ), $\Sigma_{1}$ is involutive in the $x^{2}$-direction and its secondary system is hyperbolic.

The primary equations of $\Sigma_{1}$ may be written in the form

$$
\begin{aligned}
& \left.M^{\lambda}-j_{2 \theta-1}^{\mu} w_{\mu}^{\lambda} K^{\theta}=0, \quad \text { (summing on } \theta\right) \\
& I^{\theta}=0
\end{aligned}
$$

where $J=\left(j_{\mu}^{\lambda}\right)$. That is,

$$
\begin{gathered}
\frac{\partial z^{\lambda}}{\partial x^{2}}-A_{\mu}^{\lambda} \frac{\partial z^{\mu}}{\partial x^{1}}+w_{\mu}^{\lambda} j_{2 \theta-1}^{\mu} v^{2 \nu-1} \frac{\partial z^{\nu}}{\partial x^{1}} \\
-j_{2 \theta-1}^{\mu} w_{\mu}^{\lambda} u^{\theta}-B^{\lambda}=0 .
\end{gathered}
$$

Since

$$
\begin{aligned}
& w_{\lambda}^{\mu}\left(A_{\mu}^{\nu}-j_{\omega \theta-1}^{\omega} w_{2}^{\nu} v_{\mu}^{2 \theta-1}\right) u_{\nu}^{\pi} \\
& \quad=j_{\bar{\lambda}}^{\pi}-\delta_{\lambda}^{2 \theta-1} j_{2 \theta-1}^{\omega} \delta_{\omega}^{\pi}=j_{\hat{\lambda}}^{\pi}-\delta_{\lambda}^{2 \theta-1} j_{2 \theta-1}^{\bar{\sigma}} \\
& \quad=\left\{\begin{array}{cc}
j_{\lambda}^{\pi} \text { if } \lambda \neq 2 \varphi-1, & 1 \leqq \varphi \leqq r \\
0 \text { if } \lambda=2 \varphi-1, & 1 \leqq \varphi \leqq r,
\end{array}\right.
\end{aligned}
$$

the relevant matrix for $\Sigma_{1}$ is diagonalized.

CoRollary. For a system satisfying the conditions of Theorem 5 the initial value problem is well posed.

The author would like to thank J. Jans for helpful discussions of the algebraic problems in this paper. 


\section{REFERENCES}

1. R. Hermann, E. Cartan's Geometric Theory of Partial Differential Equations, Advances in Mathematics, 1 (1965), 265-317.

2. H. H. Johnson, Absolute equivalence of exterior differential systems, (to appear in Illinois J. Math. 10 (1966), 407-411

3. P. Lax, Partial differential equations, Notes, NYU (1950-51).

4. L. Mansfield, A generalization of the Cartan-Kahler theorem, Thesis, 1965, University of Washington.

Received July 11, 1966.

UNIVERSITY OF WASHINGTON

Seattle, Washington 


\section{PACIFIC JOURNAL OF MATHEMATICS}

\section{EDITORS}

\section{H. SAMELSON}

Stanford University

Stanford, California

J. P. JANS

University of Washington

Seattle, Washington 98105
J. DUGUNDJI

University of Southern California Los Angeles, California 90007

RICHARD ARENS

University of California

Los Angeles, California 90024

\section{ASSOCIATE EDITORS}
E. F. BECKENBACH
B. H. NEumanN
F. WOLF
K. YosidA

\section{SUPPORTING INSTITUTIONS}

\author{
UNIVERSITY OF BRITISH COLUMBIA \\ CALIFORNIA INSTITUTE OF TECHNOLOGY \\ UNIVERSITY OF CALIFORNIA \\ MONTANA STATE UNIVERSITY \\ UNIVERSITY OF NEVADA \\ NEW MEXICO STATE UNIVERSITY \\ OREGON STATE UNIVERSITY \\ UNIVERSITY OF OREGON \\ OSAKA UNIVERSITY \\ UNIVERSITY OF SOUTHERN CALIFORNIA
}

\author{
STANFORD UNIVERSITY \\ UNIVERSITY OF TOKYO \\ UNIVERSITY OF UTAH \\ WASHINGTON STATE UNIVERSITY \\ UNIVERSITY OF WASHINGTON \\ AMERICAN MATHEMATICAL SOCIETY \\ CHEVRON RESEARCH CORPORATION \\ TRW SYSTEMS \\ NAVAL ORDNANCE TEST STATION
}

Mathematical papers intended for publication in the Pacific Journal of Mathematics should be typewritten (double spaced). The first paragraph or two must be capable of being used separately as a synopsis of the entire paper. It should not contain references to the bibliography. Manuscripts may be sent to any one of the four editors. All other communications to the editors should be addressed to the managing editor, Richard Arens at the University of California, Los Angeles, California 90024.

50 reprints per author of each article are furnished free of charge; additional copies may be obtained at cost in multiples of 50 .

The Pacific Journal of Mathematics is published monthly. Effective with Volume 16 the price per volume ( 3 numbers) is $\$ 8.00$; single issues, $\$ 3.00$. Special price for current issues to individual faculty members of supporting institutions and to individual members of the American Mathematical Society: $\$ 4.00$ per volume; single issues $\$ 1.50$. Back numbers are available.

Subscriptions, orders for back numbers, and changes of address should be sent to Pacific Journal of Mathematics, 103 Highland Boulevard, Berkeley 8, California.

Printed at Kokusai Bunken Insatsusha (International Academic Printing Co., Ltd.), 7-17, Fujimi 2-chome, Chiyoda-ku, Tokyo, Japan.

PUBLISHED BY PACIFIC JOURNAL OF MATHEMATICS, A NON-PROFIT CORPORATION

The Supporting Institutions listed above contribute to the cost of publication of this Journal, but they are not owners or publishers and have no responsibility for its content or policies. 


\section{Pacific Journal of Mathematics

Vol. 22, No. $3 \quad$ March, 1967

Wai-Mee Ching and James Sai-Wing Wong, Multipliers and $H^{*}$

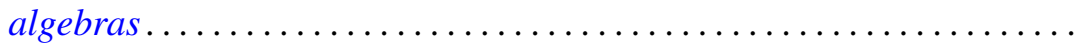

P. H. Doyle, III and John Gilbert Hocking, A generalization of the Wilder

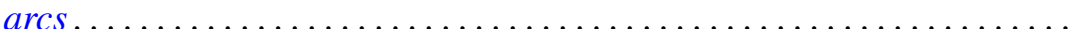

Irving Leonard Glicksberg, A Phragmén-Lindelöf theorem for function

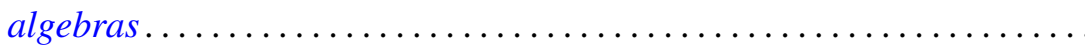

E. M. Horadam, A sum of a certain divisor function for arithmetical semi-groups ..................................... 407

V. Istrăţescu, On some hyponormal operators ................... 413

Harold H. Johnson, The non-invariance of hyperbolicity in partial

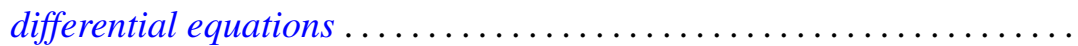

Daniel Paul Maki, On constructing distribution functions: A bounded denumerable spectrum with $n$ limit points................... 431

Ronald John Nunke, On the structure of Tor. II .................... 453

T. V. Panchapagesan, Unitary operators in Banach spaces ............. 465

Gerald H. Ryder, Boundary value problems for a class of nonlinear differential equations ................................. 477

Stephen Simons, The iterated limit condition and sequential convergence .................................... 505

Larry Eugene Snyder, Stolz angle convergence in metric spaces ......... 515 Sherman K. Stein, Factoring by subsets ................... 523

Ponnaluri Suryanarayana, The higher order differentiability of solutions of abstract evolution equations . . .

Leroy J. Warren and Henry Gilbert Bray, On the square-freeness of Fermat and Mersenne numbers ............................... 563

Tudor Zamfirescu, On l-simplicial convexity in vector spaces........... 565 Eduardo H. Zarantonello, The closure of the numerical range contains the spectrum 